\title{
The Significance of the Study about the Biological Effects of Solar Ultraviolet Radiation using the Exposed Facility on the International Space Station
}

\author{
Akihisa Takahashi and Takeo Ohnishi
}

Department of Biology, Nara Medical University School of Medicine, Shijo-cho 840, Kashihara, Nara 634-8521, Japan

\begin{abstract}
It is believed that ultraviolet (UV) radiation from the sun participated in events related to the chemical evolution and birth of life on the primitive Earth. Although UV radiation would be also a driving force for the biological evolution of life on Earth, life space of the primitive living organisms would be limited in the UV-shielded place such as in the water at an early stage of the evolution of life. After the formation of stratospheric ozone layer through the production of oxygen by photoautotroph, living organisms were able to expand their domain from water to land. As a result, now, many kinds of living organisms containing human beings are flourishing on the ground. In the near future, increased transmission of harmful solar UV radiation may reach the Earth's surface due to stratospheric ozone layer depletion. In order to learn more about the biological effects of solar UV radiation with or without interruption by the ozone layer, the utilization of an Exposed Facility on the International Space Station is required. Experiments proposed for this facility would provide a tool for the scientific investigation of processes involved in the birth and evolution of life on Earth, and could also demonstrate the importance of protecting the Earth's future environment from future ozone layer depletion.
\end{abstract}

Key words: Exposed Facility, ISS, UV, ozone layer.

\section{Solar ultraviolet $(U V)$ radiation and the birth of life on Earth}

Since Miller's experiments (Miller and Urey, 1959), many kinds of organic compounds are commonly believed to have been produced by solar UV radiation at an early stage in the chemical evolution and birth of life on Earth about 4 billion years ago. At that time, the atmosphere is believed to have been anaerobic without an ozone layer. Therefore, solar UV radiation was the most important source of energy on the primitive Earth when compared to the present. At the same time, the presence of such strong solar UV radiation in the environment was also likely to have produced harmful side effects on early life on Earth. UV radiation leads to DNA damage such as the formation of pyrimidine dimers by inducing covalent linkages between adjacent pyrimidines on DNA molecules. The two major classes of DNA lesions induced by UV (UVC, 200-290 nm; UVB, 290-320 nm) are cis-syn cyclobutane-pyrimidine dimers (CPDs) and pyrimidine (6-4) pyrimidone photoproducts (6-4PPs). 6 -4 PPs are formed at a frequency of $20-30 \%$ of the yields of CPDs (Friedberg et al., 1995). These photoproducts block RNA and DNA synthesis. DNA damage, when unrepaired, can contribute to mutagenesis, developmental

Review

Received January 4, 2005

Address for correspondence: Dr. Takeo Ohnishi Department of Biology,

Nara Medical University School of Medicine, Shijo-cho 840, Kashihara, Nara 634-8521, Japan E-mail: tohnishi@naramed-u.ac.jp abnormality, senescence and cellular lethality (Fig. 1).

\section{Survival strategies against solar $U V$}

After the beginning of life in the water, it has been speculated that the newly developing organisms would have found it difficult to survive on land due to the large amount of solar UV radiation reaching the Earth's surface. The intense short wavelength radiation would have been especially harmful. Multiple survival strategies and biological mechanisms would have evolved in order to minimize the genotoxic consequences of this harmful UV radiation for the early stages of evolution of life on our planet. Early survival strategies for living organisms would have included the development of biological systems capable of protecting the organisms from UV radiation (Fig. 1). It is well known that most organisms synthesize chemical compounds capable of absorbing UV radiation, and thus decrease the formation of DNA damage inflicted by harmful solar UV radiation. Mycosporine-like amino acids (MAAs) are the most important UV-absorbing pigments in primitive aquatic and land organisms (e.g. cyanobacteria, algae and lichens)

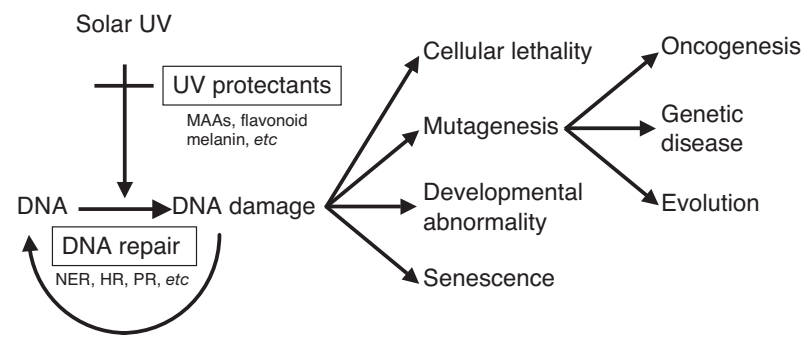

Fig. 1. Biological effects of solar UV radiation. 
(Björn et al., 2002; Rozema et al., 2002). Mycosporineglycine, one of the MAAs, is very good protecting from the critical spectral region such as UVB. Charophyes of the ancestor of land plants had already acquired a new biosynthetic pathway of aromatic-compounds such as sikimate pathway. It also made possible the synthesis of more efficient UV-absorbing pigments such as flavonoids, which strongly absorb a wide range of UV wavelengths (230-380 nm). Direct evidence showing that flavonoids play a role in protecting cells against UVinduced damage has been reported (Takahashi et al., 1991). Flavonoids occur in primitive land organisms (e.g. mosses and pteridophytes) as well as higher land plant (Björn et al., 2002). Flavonoids accumulate primarily in the vacuoles of epidermal cells in vascular plants after exposure to near UV wavelengths. It has been reported that a key enzyme involved in flavonoid synthesis might be chalcone synthase, and this system can help protect organisms from UV light thus permitting increased survival rates, which could have aided development and evolution (Yamazaki et al., 2001).

In animals, melanin can absorb UV efficiently at most wavelengths and help protect organisms from UV radiation. An important consideration is whether the protective benefits of melanin derive solely from its function as an optical filter. The absolute amount of melanin in the skin is an important factor, but its distribution in humans plays an important a role in determining visible skin color and UV absorption. It has been reported that melanin does afford significant protection against DNA damage (Kobayashi et al., 1993).

Another survival strategy to protect organisms against UV-induced DNA damage is the presence of DNA repair systems (Fig. 1). It is well known that most organisms possess DNA repair systems to protect themselves from harmful UV. There are several UV repair systems in most organisms. First, there are photoreactivation (PR) mechanisms. PR is dependent on just one enzyme, DNA photolyase (Sancar, 1994). DNA photolyase repairs DNA by utilizing the energy of blue, violet or longwave UV light to break the covalent linkages of the UV generated CPDs or 6-4PPs (Todo et al., 1993). Although the PR mechanisms are absent in placental mammals (e.g. humans and mice), photolyases exist in bacteria, lower eukaryotes, plants and many animals including marsupials. PR may have been an indispensable repair system for life exposed to the harmful solar UV radiation before it had moved onto land. The second repair pathway utilizes nucleotide excision repair (NER). NER proceeds through a number of discrete enzymatic steps (Hanawalt, 2002). These steps include the recognition of DNA damage; the introduction of incisions into the damaged DNA strand with an incision made on each side of the UV induced DNA lesion; removal of the DNA oligonucleotide containing the lesion; resynthesis of the deleted nucleotide sequence using the complementary DNA strand as a template; and finally, ligation of the newly-synthesized repair patch to the pre-existing DNA strand. This complete series of reactions can be reconstituted in vitro (Sancar, 1996). For a cell to survive UV damage, the blocked DNA polymerase must guide the NER machinery to the site of the lesion, which is then repaired in an error-free manner. Finally, the third repair pathway utilizes homologous recombination repair (HR). HR and NER seem to be universally present in most organisms. This can function through at least two pathways: a Rad51-dependent pathway, which is predominantly error free, or a Rad51-independent pathway, which is error prone (Griffin and Thacker, 2004). HR also proceeds through a number of discrete enzymatic steps. In theory, chromosome exchanges can result from repair or mis-repair using either pathway, error free or error prone.

\section{$U V$ radiation as a driver for the evolution of life on Earth}

Biological evolution consists of the generation of heritable variability and the differential survival of the resulting offspring (Darwin, 1859). UV radiation has a dual role in evolution as both a potent mutagen and selective agent. DNA repair is somehow coupled to transcription and this strongly influences the mutation spectrum induced by UV radiation. This means that repair is more efficient or shows a preference for the transcribed DNA strand which results in reducing the number of base substitutions or mutations, which arise on the active or transcribed DNA strand relative to the untranscribed DNA strand. The replicative DNA polymerase complex may be blocked as a result of UV damage, and in this situation, lesion bypass can be accomplished using error-free mechanisms or using error-prone mechanisms, which involves the newly described, non-processive DNA polymerase $\zeta$ and Rev1 (Lawrence, 2002). Understanding this process is likely to provide information relevant to the origin of oncogenesis, genetic disease, and evolution. It would appear that solar UV radiation might have been a prime motivational force for the evolution and development of these important repair mechanisms (Fig. 1). In addition, $\mathrm{UV}$ radiation may have been driving force in the origin and the evolution of sexual processes, because there is experimental evidence linking UV damage repair and sexual processes (Mongold, 1992).

The most important role of sunlight in evolution has been as a source of energy for photosynthesis. At an early stage of the evolution of life, about 2.7 billion years ago, cyanobacterium with photosynthetic activity arose using the visible light penetrated into water. The atmosphere on the Earth might have been gradually altered from an anaerobic to an aerobic condition ever since. It is believed that the environmental change such as aerobic condition finally triggered the Cambrian animal explosion (Ohno, 1997). Once oxygen was present, solar UV radiation could have generated ozone from oxygen. 


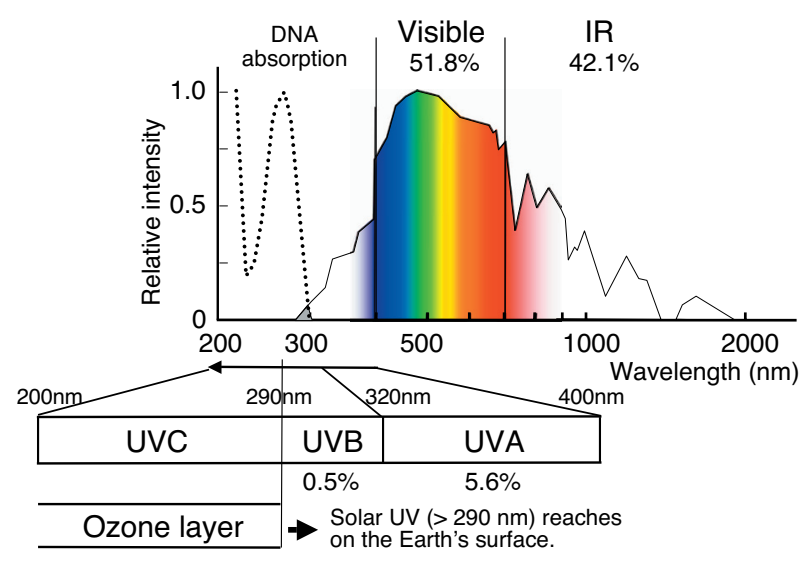

Fig. 2. Spectrum of solar radiation at the Earth's surface.

Ozone molecules consist of three oxygen atoms. In the atmosphere, at an altitude between 10 and $50 \mathrm{~km}$, the ozone layer is present in trace amounts at a part per million level. If the whole atmosphere were condensed down to sea-level pressure and room temperature, the ozone would form a layer with a thickness of about 3.2 $\mathrm{mm}$. Figure 2 shows the mean quantities of the solar spectral radiation reaching the Earth's surface (Jankowski and Cader, 1997). The proportion of the solar UV radiation currently reaching the Earth's surface is only 6.1 $\%$. Although the atmosphere does not absorb in the UVA region (wavelengths from 320 to $400 \mathrm{~nm}$ ), ozone and oxygen completely absorb radiation in the UVC region $(<$ $290 \mathrm{~nm}$ ), so no radiation at these wavelengths reaches the Earth's surface. After the formation of the ozone layer about 0.4 billion years ago, living organisms were able to expand their domain from water to land because the ozone layer provided a filter for the protection of living organisms from UV radiation.

\section{The biological effect of depletion of the stratospheric ozone layer}

Human activities, including the production of chlorofluorocarbons, have generated numerous chemicals and gases which have migrated into the upper parts of the Earth's atmosphere and which have disturbed the atmospheric photochemical balance, which, up to now, has stabilized the level of stratospheric ozone. An important consequence of the depletion of stratospheric ozone is the increased transmission of solar UVB radiation (290-320 $\mathrm{nm}$ ) to the Earth's surface. The discovery of the ozone 'hole' in 1980s was a big shock in the world. It took 11 years of assessment, research, and negotiations to promote the first general ozone agreement in 1985. This agreement, known as the Vienna Convention for the Protection of the Ozone Layer, was a pledge to protect the ozone layer. In order to reduce the production and release of ozone depleting substances such as chlorofluorocarbons, specific commitments were agreed to in 1987 in the Montreal Protocol (UNEP homepage, 2004). A striking biological observation is that DNA absorbs strongly in the UVB region, with an absorption spectrum paralleling that of ozone; it is as if ozone provides a perfect umbrella to shield biological matter from the solar UV radiation. Figure 3 shows the absorption spectra for DNA, the solar flux at the Earth' s surface under a normal atmosphere, and the projected absorption spectra for the situation in which the ozone layer has been deleted by $30 \%$ (Lloyd, 1993). The biological effectiveness of UV is calculated from its overlap with the DNA absorption spectrum (Fig. 3c) which is considered as the appropriate action spectrum to enable or predict certain phenomena. When the ozone abundance is reduced $30 \%$ (Fig. $3 a$ and $3 b$ ), calculations predict an increase of about $200 \%$ increase in the biological effectiveness of incident UV radiation (Fig. $3 d$ and $3 e$ ). In fact, it was reported that a 1, 10, 30 and 50\% reduction in ozone levels would result in a 2, 24, 200 and $400 \%$ increase in the frequencies of non-melanoma skin cancers, respectively (Setlow, 1974). At larger ozone losses, the overlap between the UV action spectrum and the enhanced solar flux begins to increase exponentially.

The harmful effects of UV on live organisms constitute a danger for the health of the entire human population. There is no doubt that cumulative exposure to UV is important in the etiology of skin cancer (Setlow, 1974), cataract formation (Oriowo et al., 2001), and has effects on the immune system (Jeevan and Kripke, 1993). It is also known that an increase of UV radiation at the Earth's surface could cause severe ecological problems through the effect that UV would have on important living organisms such as phytoplankton (Smith et al., 1992) and plants (Teramura, 1983; Paul, 2000; Rousseaux et al., 2004) which are continuously exposed to solar UV radiation during the day.

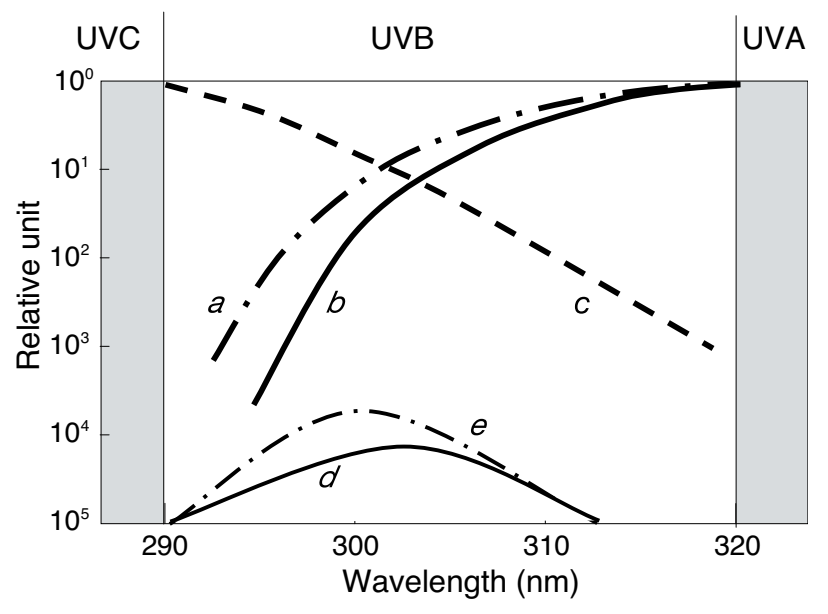

Fig. 3. Solar irradiance shielding by the ozone layer for: $a$ and $b$, solar irradiance; $c$, DNA absorption; $d$ and $e$, biological effectiveness. $a$ and $e$; $30 \%$ ozone reduction. $b$ and $d$, with the present ozone layer. 


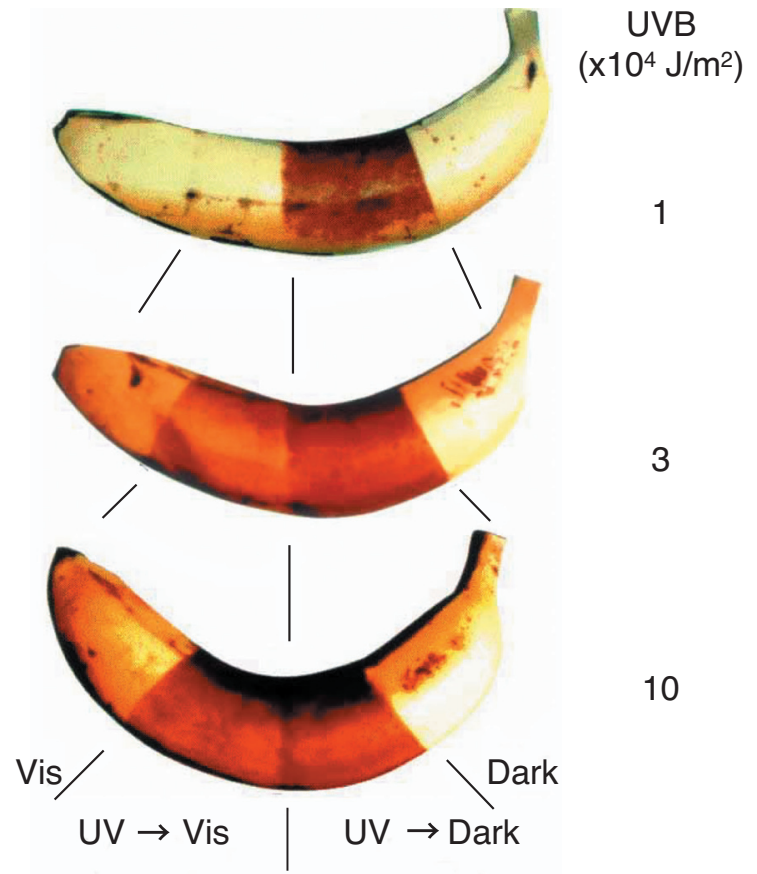

Fig. 4. Limitations of photoreactivation in the banana. Bananas were irradiated with a health lamp (HL; FL20SE, Toshiba, Tokyo, Japan), which emits light with wavelengths longer than $270 \mathrm{~nm}$ and contains mainly UVB (peak at $315 \mathrm{~nm}$ ) with a Kodacel filter (cuts off radiation wavelengths less than $290 \mathrm{~nm}$ ) (Richard, 1979). The intensity of HL light was determined with a UV digital radiometer (UV103, Macam photometrics Ltd, Livingston, Scotland). After exposure to radiation with an HL light, the bananas were kept at $25^{\circ} \mathrm{C}$ for 2 days. A part of the banana was irradiated with (UV $\rightarrow$ Vis) or without (UV $\rightarrow$ Dark) white light (FL20SW, Toshiba; $10 \mathrm{~cm}$ height). For controls, sections of the banana were irradiated under the same conditions with (Vis) or without (Dark) white light.

\section{Limitations of PR activity after solar $U V$ exposure}

To examine the limits of PR activity in protecting organisms from solar UV radiation, PR activity was studied in the banana (Ohnishi et al., 2002). UVB irradiation induced blackening and white light induced $\mathrm{PR}$ repair in the banana. These results showed that the blackening of the banana was induced by irradiation with UVC (254 nm) and it was inhibited by PR immediately after UVC exposure (Björn, 1973). The blackening of the banana was dependent on the fluence of UVB (Fig. $4, \mathrm{UV} \rightarrow$ dark). In addition, the blackening caused by irradiation at $10^{4} \mathrm{~J} / \mathrm{m}^{2}$ was inhibited by irradiation with white light immediately after UVB irradiation (Fig. 4, $\mathrm{UV} \rightarrow$ Vis). Since bananas displayed PR activity in the UVB-irradiated portions of their skin, it is suggested that the blackening of the skin was induced as a result of the formation of pyrimidine dimers produced by UVB on DNA molecules (Okaichi et al., 1992). In contrast, PR induced by white light was clearly detected in the bananas irradiated with UVB at a dose rate of less than 3 $\mathrm{x} 10^{4} \mathrm{~J} / \mathrm{m}^{2}$, it was not detected in bananas irradiated with UVB at $10^{5} \mathrm{~J} / \mathrm{m}^{2}$.
These results suggest that bananas do not have a sufficient PR capacity for complete recovery if there are too many UVB-induced lesions; these experimental results suggest that the PR system in the banana is limited in the amount or extent of UV damage repair it can repair.

\section{An outline of the space biological experiments proposed for an Exposed Facility}

The International Space Station (ISS), a large-scale multinational project, began with the first assembly mission in November 1998. The ISS circles the Earth at approximately $400 \mathrm{~km}$ over the ozone layer. An Exposed Facility (EF) will be an area available for longterm experiments in open space, as well as for Earth and astronomical observations (JAXA homepage, 2004). The $\mathrm{EF}$ is a unique facility in that it will enable astronauts to conduct experiments with direct exposure to space without any shielding or protection. One of the most important projects planned for the EF is the observation of the Earth's environment. The EF will permit studies of many problems from space, and provide observations and information to help solve them. Scientists will be able to study the presence of trace gases that deplete the ozone layer, and observe short wavelength radiation emitted by ozone. In addition, the world's largest wideangle X-ray camera will be mounted on the platform to make observations of space, examine phenomena beyond our galaxy, and refine our map of the distribution of the galaxies.

Although the planned experiments for astronomy and physics are well defined, the biological experiments utilizing the properties of the EF on the ISS have yet to be completely designed. The EF will be exposed directly to solar UV radiation without the interruption of an ozone layer. Therefore, the EF could thus serve as a model for UV radiation exposure in the atmosphere present on the primitive Earth's surface, and also as a model for future solar radiation exposure at the earth's surface under a depleted ozone layer. Spores of Bacillus subtilis were exposed to a space environment, and their survival was analyzed after their retrieval from a space facility (Facius et al., 1978; Horneck et al., 1984; Horneck et al., 1994; Horneck et al., 1995; Rettberg et al., 2002). Although the bacteria were exposed to cosmic radiation in space, solar UV was found to be the most damaging radiation present. By using different thicknesses and arrangements of filters, solar particles containing solar UV and space radiation can be filtered to produce exposures with the desired quantity and quality of radiation for experiments (Table 1). In the proposal described here, it is suggested that the EF on the ISS is an ideal platform to observe various important biological end-points in bacteria, cells, and small organisms in response to shielded and unshielded solar UV radiation. The endpoints to be examined and studied would be DNA damage, mutation, repair, transformation, $u m u$-induction, survival, growth 
Miller, S.L. and Urey, H.C. (1959) Organic compound synthesis on the primitive earth. Science, 130, 245-251.

Mongold, J.A. (1992) DNA repair and the evolution of transformation in Haemophilus influenzae. Genetics, 132, 893-898.

Ohnishi, T., Takahashi, A. and Ohnishi, K. (2002) Studies about space radiation promote new fields in radiation biology. $J$. Radiat. Res., 43, Suppl., S261-S264.

Ohno, S. (1997) The reason for as well as the consequence of the Cambrian explosion in animal evolution. J. Mol. Evol., 44, S23-S27.

Okaichi, K., Nagashima, K., Nozu, K. and Ohnishi, T. (1992) Mutagenic specificity in DNA base sequence by irradiation of health lamp light (UV-B) in Escherichia coli. Mutat. Res., 282, 183-190.

Oriowo, O.M., Cullen, A.P., Chou, B.R. and Sivak, J.G. (2001) Action spectrum and recovery for in vitro UV-induced cataract using whole lenses. Invest. Ophthalmol. Vis. Sci., 42, 2596-2602.

Paul, N.D. (2000) Stratospheric ozone depletion, UV-B radiation and crop disease. Environ. Pollut., 108, 343-355.

Richard, M.K. (1979) Cut off filters for the near ultraviolet. Photochem. Photobiol., 29, 1053-1054.

Rettberg, P., Eschweiler, U., Strauch, K., Reitz, G., Horneck, G., Wanke, H., Brack, A. and Barbier, B. (2002) Survival of microorganisms in space protected by meteorite material: results of the experiment 'EXOBIOLOGIE' of the PERSEUS mission. Adv. Space Res., 30, 1539-1545.

Rozema, J., Björn, L.O., Bornman, J.F., Gaberščik, A., Häder, D.P., Trošt, T., Germ, M., Klisch, M., Gröniger, A., Sinha, R.P., Lebert, M., He, Y.Y., Buffoni-Hall, R., de Bakker, N.V., van de Staaij, J. and Meijkamp, B.B. (2002) The role of UV-B radiation in aquatic and terrestrial ecosystems-an experimental and functional analysis of the evolution of UV-absorbing compounds. J. Photochem. Photobiol. B, 66, $2-12$

Rousseaux, M.C., Flint, S.D., Searles, P.S. and Caldwell, M.M. (2004) Plant responses to current solar ultraviolet-B radiation and to supplemented solar ultraviolet-B radiation simulating ozone depletion: an experimental comparison paragraph sign. Photochem. Photobiol., 80, 224-230.

Sancar, A. (1994) Structure and function of DNA photolyase. Biochemistry, 33, 2-9.

Sancar, A. (1996) DNA excision repair. Annu. Rev. Biochem., 65, 43-81.

Setlow, R.B. (1974) The wavelengths in sunlight effective in production skin cancer: a theoretical analysis. Proc. Natl. Acad. Sci. U.S.A., 71, 3363-3366.

Smith, R.C., Prezelin, B.B., Baker, K.S., Bidigare, R.R., Boucher, N.P., Coley, T., Karentz, D., MacIntyre, S., Matlick, H.A., Menzies, D., Ondrusek, M., Wan, Z. and Waters, K.J. (1992) Ozone depletion: ultraviolet radiation and phytoplankton biology in antarctic waters. Science, $\mathbf{2 5 5}$, 952-959.

Takahashi, A., Takeda, K. and Ohnishi, T. (1991) Light-induced anthocyanin reduces the extent of damage to DNA in UVirradiated Centaurea cyanus cells in culture. Plant Cell Phys., 32, 541-547.
Teramura, A.H. (1983) Effects of ultraviolet-B radiation on the growth and yield of crop plants. Physiol. Plant, 58, 415-427.

Todo, T., Takemori, H., Ryo, H., Ihara, M., Matsunaga, T., Nikaido, O., Sato, K. and Nomura, T. (1993) A new photoreactivating enzyme that specifically repairs ultraviolet light-induced (6-4)photoproducts. Nature, 361, 371-374.

UNEP homepage (2004) http://www.unep.ch/ozone/ Public_Information/index.asp.

Yamazaki, Y., Suh, D.Y., Sitthithaworn, W., Ishiguro, K., Kobayashi, Y., Shibuya, M., Ebizuka, Y. and Sankawa, U. (2001) Diverse chalcone synthase superfamily enzymes from the most primitive vascular plant, Psilotum nudum. Planta, 214, 75-84. 